\title{
FIELD CONCENTRATION NEAR CIRCULAR CONDUCTORS
}

\section{H. PORITSKY}

1. Introduction. The problem considered in this note is the calculation of the maximum field concentration which obtains in the field shown in Figure 1a. Here the semicircles represent the upper half of an infinite row of cylindrical metallic conductcrs at a constant potential, the conductors touching each other. There is a potential difference between these and the upper plane surface, and it is required to calculate the resulting field concentration which obtains at

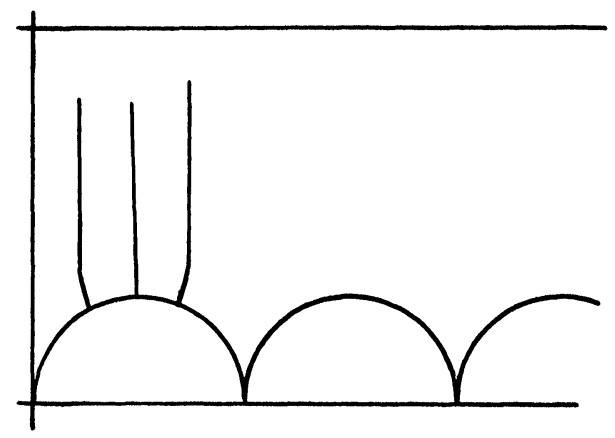

Fig. 1a

the semicircular conductors. The maximum electric gradient will obviously occur at the midpoints between the points of contact with the adjacent conductors.

It is assumed that the plane surface representing the other electrode is sufficiently far away so that the field is quite uniform near it and the field concentration is not affected by the distance between the circular wires and the plane surface. In fact, throughout the following it will be supposed that this plane is at infinity.

The above problem is of interest in the construction of electric cable where a row of circular wires is of ten arranged around a circular cylindrical surface and a potential difference main tained between the ions and an outer circular sheath concentric with the axis of the cylinder and external to the wires. To the first approximation the field concentration factor which obtains in Figure 1a may be carried over to the case of a cable by first assuming that the surface made up

Received by the editors August 17, 1942. 
of the semicircles is replaced by a tangent cylinder, calculating the resulting electric gradient at this surface, and then multiplying it by the field concentration factor derived from the plane case.

2. Solutions by means of the modular function. The method employed in this note utilizes the modular function, $\lambda(\tau)$, and is based upon the fact that the function just mentioned maps the shaded region of Figure $1 \mathrm{~b}$ in the $\tau$-plane upon the shaded region of the $\lambda$-plane

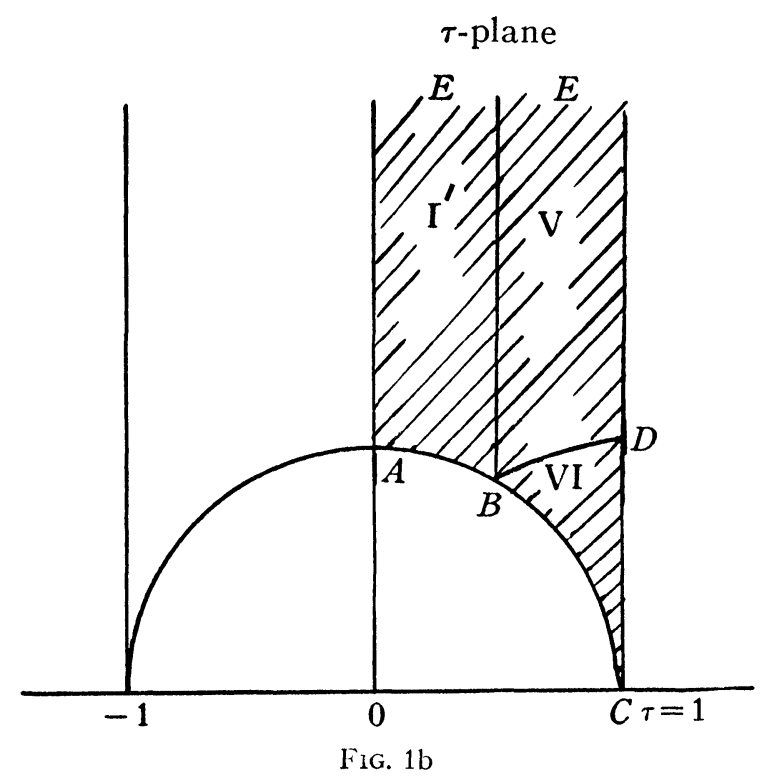

shown in Figure 1c (see Burkhardt, Elliptische Funktionen, Berlin, 1920 , p. 259). Thus the rather complicated boundary consisting partly of circles and partly of straight lines is now replaced by rectilinear boundaries only. Therefore the solution of the problem can be carried out very simply. The analytic expression for $w$ in terms of $\lambda$ is given below.

It will be noticed that the $\tau$-plane is simply related to the original $z$-plane. In fact, this relation is given by

$$
z=(\dot{\pi} / 2) \tau .
$$

If the region to the left of the pure imaginary axis of Figure $1 \mathrm{~b}$, and forming the mirror image of the shaded region, is added to the latter, the combined region which is bounded by the complete semicircle from $\tau=-1$ to $\tau=1$ and two vertical lines through $\tau=1$, 
$\tau=-1$ goes into a half-plane in Figure 1c, namely the half-plane to the left of the vertical axis through $\lambda=1 / 2$. Since the flux lines in Figure 2 all meet the circular boundary normally, it follows that the corresponding flux lines of Figure 1c will all cross the vertical straight line through $\lambda=1 / 2$ normally. In Figure $1 \mathrm{~b}$ the flux lines go off to infinity; in Figure 1c they must converge toward the origin $E$, which

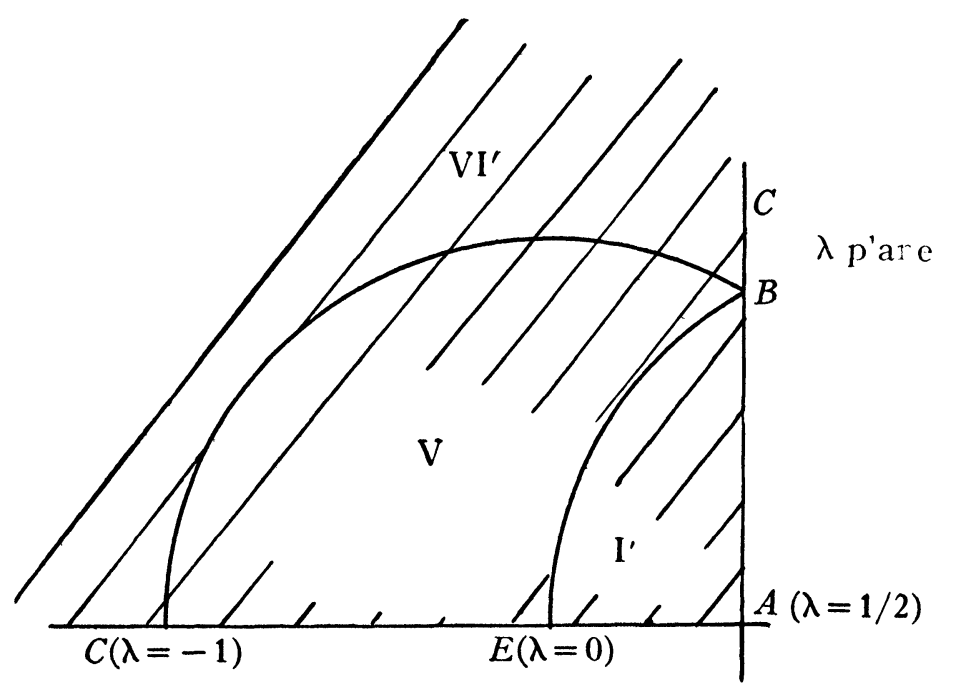

FIG. 1c

point corresponds to the infinite region of Figure $1 \mathrm{~b}$, at least for ways of recession to infinity in the direction of the pure imaginary axis in the $\tau$-plane. This means that the flux lines in Figure 1c are simply due to positive and negative point charges placed, respectively, at the origin $\lambda=0$ and at the point $\lambda=1$, which is its negative image in the vertical line through $\lambda=1 / 2$ (that is, through $A$ of Figure 1c). Therefore one might at once express the flux function in terms of $\lambda$ as follows:

$$
w=(1 / 2) \ln \frac{\lambda-1}{\lambda} .
$$

The factor $1 / 2$ is due to the fact that $\pi$ flux lines are supposed to converge toward the origin $\lambda=0$, since $\pi$ flux lines receded to infinity in the original $z$-plane for each period strip.

3. Expansions in powers of $q$. The expression for the modular function is given by 


$$
\lambda=\lambda(\tau)=\frac{16 q\left(1+q^{1 \cdot 2}+q^{2 \cdot 3}+\cdots\right)^{4}}{\left(1+2 q+2 q^{4}+2 q^{9}+\cdots\right)^{4}}
$$

(see Burkhardt, loc. cit. p. 253), where $q$ is given by

$$
q=e^{\tau \pi i} \text {. }
$$

It will be recalled that this modular function is of interest in connection with transformation of periods of an elliptic function and that the expression obtained for it, namely equation (3) above, can be derived from the $\vartheta$-functions, being in fact equal to

$$
\lambda(\tau)=\frac{\vartheta_{2}^{4}(0)}{\vartheta_{3}^{4}(0)} ;
$$

the $\vartheta$ 's are periodic functions of interest in the theory of elliptic functions and involve as a parameter the quantity $q$, though this has not been indicated in the notation of the right-hand side of (5). $q$ itself is given by (4) where $\tau$ is the ratio of the two periods of the elliptic function in question. This connection of the function $\lambda(\tau)$ with elliptic functions is not utilized in the following, and is mentioned here only in passing. The only essential feature is the fact that the function $\lambda(\tau)$ defined by (3) and (4) maps the shaded region of Figure $1 \mathrm{~b}$ upon that of Figure 1c, and yields a simple expression for $w$ (equation (2)).

To proceed with the calculation of the field it is now necessary to evaluate the expression of the derivative $d w / d z$ whose absolute value gives the field strength at any point. This derivative will be evaluated as a product of four distinct derivatives as follows:

$$
\frac{d w}{d z}=\frac{d w}{d \lambda} \frac{d \lambda}{d q} \frac{d q}{d \tau} \frac{d \tau}{d z} .
$$

It is clear from (3) that it is easier to differentiate the logarithm of $\lambda$ rather than $\lambda$ itself; hence we shall utilize this derivative:

$$
\begin{aligned}
\frac{d \log \lambda}{d q}=\frac{1}{\lambda} \frac{d \lambda}{d q}=\frac{1}{q}+ & 4 \frac{1 \cdot 2 q+2 \cdot 3 q^{5}+\cdots}{1+q^{1 \cdot 2}+q^{2 \cdot 3}+\cdots} \\
& -4 \frac{1 \cdot 2 q+2 \cdot 3 q^{5}+\cdots}{1+2 q+2 q^{4}+2 q^{9}+\cdots}
\end{aligned}
$$

Again, from (4) follows

$$
d q / d \tau=\pi i e^{\tau \pi i}=\pi i q
$$

while (1) and (2) yield 


$$
d z / d \tau=2 / \pi,
$$$$
\frac{d w}{d \lambda}=\frac{1}{2}\left[\frac{1}{\lambda-1}-\frac{1}{\lambda}\right] \text {. }
$$

Substituting from (7)-(10) into (6) there results

$$
\begin{aligned}
\frac{d w}{d z}= & \frac{1}{2}\left[\frac{1}{\lambda+1}-\frac{1}{\lambda}\right] \lambda \frac{d(\log \lambda)}{d q} n i q \frac{2}{\pi} \\
= & i\left[\frac{1}{\lambda-1}\right]\left[1+4 \frac{1 \cdot 2 q^{1 \cdot 2}+2 \cdot 3 q^{2 \cdot 3}+\cdots}{1+q^{1 \cdot 2}+q^{2 \cdot 3}+\cdots}\right. \\
& \left.-8 \frac{q+4 q^{4}+9 q^{9}+\cdots}{1+2 q+2 q^{4}+2 q^{9}+\cdots}\right] .
\end{aligned}
$$

The values of the various variables corresponding to the point $A$ of Figure $1 \mathrm{~b}$ are

$$
\tau=i, \quad \lambda=1 / 2, \quad q=e^{-\pi}=10^{\overline{2} .635624}=.043214 .
$$

Substituting in (11) and taking absolute values, there results

$$
\left|\frac{d w}{d z}\right|=2[1+.0149-.3184]=1.3952 .
$$

This leads to the field concentration factor 1.3952 .

The above served to check the field concentration factor 1.4 which was first obtained by means of a free-hand flux plot of this field shown in Figure 2.

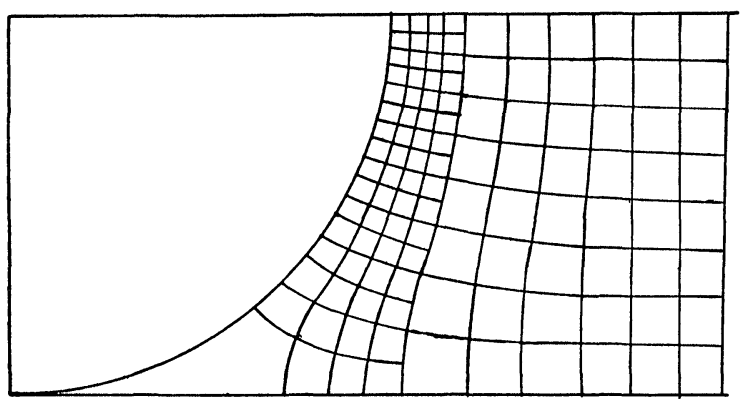

FIG. 2

General Electric Company,

SCheneCtady, N. Y. 